\title{
Communication: The key to patient satisfaction
}

\author{
Barbara Lewis* \\ Joan's Family Bill of Rights, 2934 Beverly Glen Circle, Suite 240, Los Angeles, CA 90077, USA
}

Almost everything that went wrong in the ICU, where my sister spent her last two weeks, was related to poor communication: the hospitalist, who didn't believe my research that indicated my sister should be taken off a blood thinner, which eventually she was; the nurse who dismissed my claim that something was wrong with the monitor, only to discover that my sister was "critical" when the machine was finally replaced; and the legions of clinicians who marched into her room beginning procedures or activities without a word to my terrified sister.

For nearly two decades, major U.S. medical organizations have highlighted the importance of communication skills. Analysis of government mandated patient satisfaction surveys reflects that the survey's communication dimensions drive overall scores. If we didn't know the importance of clinicians' communication skills before, now we have patient satisfaction surveys tied to money, that prove the point. Hospitals, which recognize the link between high overall scores and high communication ratings, are scrambling to embed great communication skills in their clinicians, in fact in every staff person who interacts with the patient and family.

However, research reflects the physicians' failures in mastering the communication skill set:

- Physicians may not elicit all of the patient's concerns at the outset, leading to late-arising concerns and wasted time during the encounter.

- Physicians may not address emotions or express empathy or explore the impact of disease on patients' quality of life.

- Physicians may make incorrect assumptions about our patients' health literacy.

- Physicians may not adequately assess patients' understanding of test results, treatments or plans.

- Physicians may use medical jargon that patients don't understand and don't ask about.

- Physicians may not effectively involve patients in decision-making or in obtaining informed consent.

The springboard to effective communication is listening and the ability to maintain silence while the patients describe their symptoms. Yet, research demonstrates that clinicians interrupt patients on average of 23 seconds into the patients' stories. While an artful interruption may be warranted if the patient's narrative is wandering, obtaining the entire patient story is critical to an efficient diagnosis. While certain

Copyright: (C2017 Lewis B. This is an open-access article distributed under the terms of the Creative Commons Attribution License, which permits unrestricted use, distribution, and reproduction in any medium, provided the original author and source are credited. clinicians believe that stopping the story will limit the time needed for a diagnosis, research indicates the opposite: listening to the patient's entire description accelerates the diagnosis process.

On one side of the communication spectrum is listening and at the other end is talking. Here clinicians, who have mastered empathic skills, excel in patient relationships that have partnerships at the core. Healthcare is a team sport and prominent on that team is the patient as well as family members, who are oftentimes defined by the patients.

Reams of evidence tout the value of effective communication skills. One of the most important benefits is improved medical outcomes. Research studies over the past decade highlight a wide range of benefits for the patient, which include diagnosis, shared-decision making, treatment plans and adherence to clinicians' recommendations, and lower hospital readmission rates.

Poor communication between the physician and the patient is strongly associated with U.S. malpractice litigation. According to the hospital standards of the Joint Commission, which accredits and certifies nearly 21,000 health care organizations and programs in the U.S., poor communication among healthcare providers or within healthcare teams is a leading cause for medical error.

CRICO, an insurance program, comprised of a group of companies owned by and serving the Harvard medical community, reports that from 2009 to 2013 about 30 percent of malpractice cases involved communication factors, costing in excess of $\$ 1.7$ billion in incurred losses. Over half of the cases were provider/patient communication breakdowns with one of the top factors as unsympathetic response to patient complaints.

Not only do good communication skills improve patient satisfaction scores, but communication affects provider satisfaction, as well. According to research, caregivers who view their work as personcentered are more satisfied with their jobs than those whose work is not person-centered. With the high rate of burnout in healthcare, patientcentered communication skills can help reverse the trend.

Hopefully, the clinician, who believes the axiom that "I know best," has been replaced with the clinician, who listens well, who partners with patients and their families, and who treats patients as if they were beloved family members. My sister would have liked that.

Barbara Lewis, MBA, is the Managing Editor of DocCom, a nonprofit on-line communication skills learning curriculum launched in 2005, which helps clinicians and medical students become better communicators.

Correspondence to: Barbara Lewis, Joan's Family Bill of Rights, 2934 Beverly Glen Circle, Suite 240, Los Angeles, CA 90077, USA, E-mail: barbaralewis@ joansfamilybillofrights.com

Received: June 23, 2017; Accepted: July 24, 2017; Published: July 27, 2017 